\title{
In vitro activities of natural products against oral Candida isolates from denture wearers
}

\author{
Cristina Marcos-Arias, Elena Eraso, Lucila Madariaga and Guillermo Quindós
}

\begin{abstract}
Background: Candida-associated denture stomatitis is a frequent infectious disease. Treatment of this oral condition is difficult because failures and recurrences are common. The aim of this study was to test the in vitro antifungal activity of pure constituents of essentials oils.

Methods: Eight terpenic derivatives (carvacrol, farnesol, geraniol, linalool, menthol, menthone, terpinen-4-ol, and $\alpha$ terpineol), a phenylpropanoid (eugenol), a phenethyl alcohol (tyrosol) and fluconazole were evaluated against 38 Candida isolated from denture-wearers and 10 collection Candida strains by the CLSI M27-A3 broth microdilution method.

Results: Almost all the tested compounds showed antifungal activity with MIC ranges of $0.03-0.25 \%$ for eugenol and linalool, $0.03-0.12 \%$ for geraniol, $0.06-0.5 \%$ for menthol, $\alpha$-terpineol and terpinen-4-ol, $0.03-0.5 \%$ for carvacrol, and $0.06-4 \%$ for menthone. These compounds, with the exception of farnesol, menthone and tyrosol, showed important in vitro activities against the fluconazole-resistant and susceptible-dose dependent Candida isolates.

Conclusions: Carvacrol, eugenol, geraniol, linalool and terpinen-4-ol were very active in vitro against oral Candida isolates. Their fungistatic and fungicidal activities might convert them into promising alternatives for the topic treatment of oral candidiasis and denture stomatitis.
\end{abstract}

\section{Background}

Denture stomatitis is a common inflammatory reaction in denture-wearing patients, characterized by an erythematous inflammation of mucosal areas covered by dentures. Although this clinical entity is multifactorial, Candida albicans is the major etiological agent [1]. Moreover, other species of Candida, such as C. glabrata, C. tropicalis, C. krusei, C. parapsilosis and C. dubliniensis have been isolated from patients with denture stomatitis [2-4]. Different treatments have been proposed for Candida-associated denture stomatitis. However, there is a low number of antifungal agents and therapy can induce side effects, resistance and/or recurrence $[1,5]$. Thus, new therapeutic strategies are necessary and natural products can play an important role in the treatment as some of them can be included in mouthrinses or tooth pastes. Among natural products, essential oils are promising therapeutic tools for

\footnotetext{
*Correspondence: guillermo.quindos@ehu.es Laboratorio de Micología Médica, Departamento de Inmunología, Microbiología y Parasitología, Facultad de Medicina y Odontología, Universidad del País Vasco/Euskal Herriko Unibertsitatea, Bilbao, Spain
}

oral infections. These oils are complex mixtures of volatile compounds obtained from plants, such as the terpenoids, with antioxidant and antimicrobial properties against a wide range of pathogens, including Candida albicans and dermatophytes [6-9].

The aim of the current study was to investigate the in vitro activity of eight terpenic derivatives (carvacrol, farnesol, geraniol, linalool, menthol, menthone, terpinen-4ol, and $\alpha$-terpineol), a phenylpropanoid (eugenol) and a phenethyl alcohol (tyrosol) against oral Candida isolates from patients suffering from denture stomatitis.

\section{Methods}

\section{Microorganisms}

A total of 38 oral isolates were tested, including $10 \mathrm{C}$. albicans, 10 C. glabrata, 10 C. tropicalis, 5 C. guilliermondii, and 1 isolate each of C. parapsilosis, C. dubliniensis and C. krusei. The clinical isolates were randomly selected from those recovered from a prospective study in denture wearers attending the Odontology clinics at the Universidad del País Vasco/Euskal Herriko Unibertsitatea, Bilbao (Spain). Demographic and clinical

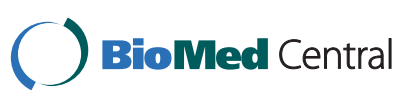


characteristics of these patients have been published elsewhere [2]. The institutional review board of Universidad del País Vasco/Euskal Herriko Unibertsitatea approved the study and informed consent was obtained in each patient included in the study. There were also studied 10 type strains from the American Type Culture Collection (ATCC) and the National Collection of Pathogenic Fungi (NCPF) including C. albicans ATCC 90028, C. albicans NCPF 3153, C. glabrata ATCC 90030, C. glabrata NCPF 3203, C. dubliniensis NCPF 3949, C. guilliermondii NCPF 3099, C. krusei ATCC 6258, C. parapsilosis ATCC 22019, C. tropicalis NCPF 3111, and the C. albicans NCPF 3153 hypha-defective mutant Ca2 (kindly donated by Professor Antonio Cassone, Istituto Superiore di Sanità, Rome, Italy). Isolates were identified by conventional mycological methods, such as the germ tube test in serum, microscopic morphology, chlamydoconidia production in corn meal agar with Tween 80, and carbon source assimilation with the commercial kit ID 32 C (bioMérieux, France) [10].

\section{Terpenes}

All evaluated compounds were purchased from Sigma (Sigma-Aldrich, USA) and included: carvacrol (5-isopropyl-2-methylphenol) from Satureja hortensis, eugenol (4-allyl-2-methoxyphenol) from Pimenta dioica, farnesol ((2E,6E)-3,7,11-trimethyldodeca-2,6,10-trien-1-ol) from Vachellia farnesiana, geraniol (3,7-dimethylocta-2,6dien-1-ol) from Rosa damascena, linalool (3,7-dimethylocta-1,6-dien-3-ol) from Coriandrum sativum, menthol $((1 R, 2 S, 5 R)$-2-isopropyl-5-methylcyclohexanol) from Mentha piperita, menthone ( $(2 S, 5 R)$-trans-2-isopropyl5-methylcyclohexanone) from Mentha piperita, terpinen-4-ol (4-isopropyl-1-methyl-1-cyclohexen-4-ol) from Melaleuca alternifolia, $\alpha$-terpineol (2-(4-methyl-1-cyclohex-3-enyl) propan-2-ol) from Artemisia annua, and tyrosol (4-(2-hydroxyethyl)phenol) from Olea europaea (Figure 1). The compounds were prepared as stock solution of 16\% (volume/volume -v/v-) in RPMI 1640 medium and $0.1 \%(\mathrm{v} / \mathrm{v})$ Tween 80 (Sigma-Aldrich) [11].

\section{In vitro antifungal activity testing}

The antifungal activities of terpenes and fluconazole (as a positive control) were determined by broth microdilution method using two fold serial dilutions in RPMI 1640 medium, as described in the document M27-A3 from the Clinical Laboratory Standards Institute (CLSI) for yeasts [12]. Briefly, chemical compounds were dissolved in RPMI 1640 medium buffered to $\mathrm{pH} 7.0$ with $0.165 \mathrm{M}$ morpholinepropanesulfonic acid. After shaking, $100 \mu \mathrm{l}$ aliquots were added to the wells of 96-well microtiter plates with final concentrations ranging from $0.01 \%$ to $8 \%(\mathrm{v} / \mathrm{v})$. To enhance the compounds solubility, Tween 80 was included in all assays at a final

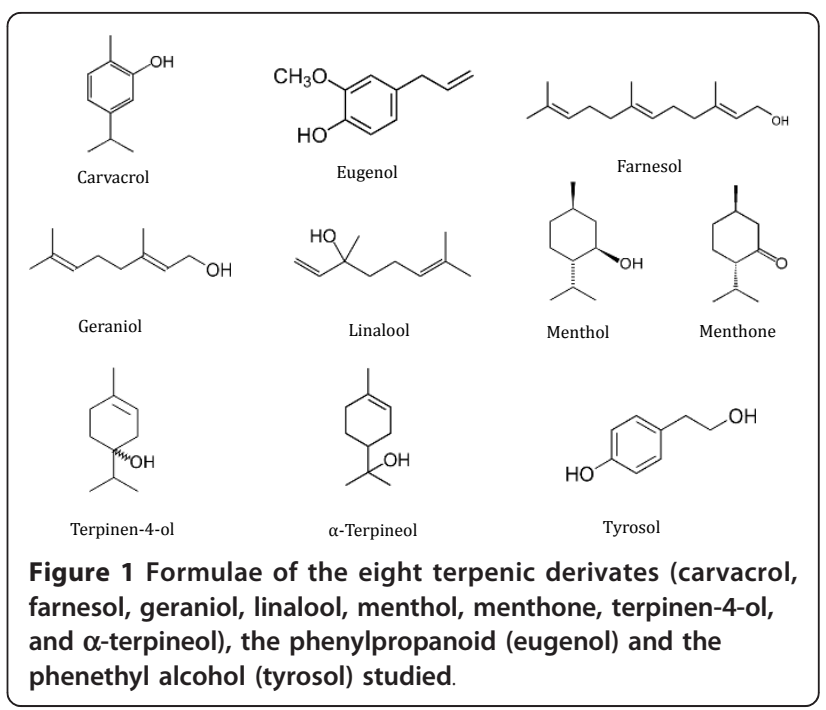

concentration of $0.05 \%(v / v)$ [11]. Fluconazole was prepared in pure water. Reference microdilution trays containing serial twofold dilutions of this drug were prepared in RPMI 1640 medium buffered to $\mathrm{pH} 7.0$ with $0.165 \mathrm{M}$ morpholinepropanesulfonic acid (MOPS) buffer in 96 -well plates, and stored at $-70^{\circ} \mathrm{C}$ for no longer than 3 months.

Yeast inocula were prepared by growing the isolates on Sabouraud dextrose agar plates for $24 \mathrm{~h}$ at $37^{\circ} \mathrm{C}$ and adjusting to a final concentration between $1 \times 10^{3}$ and 5 $\times 10^{3}$ cells $/ \mathrm{ml}$ in sterile saline. A $100 \mu \mathrm{l}$ suspension of each of the Candida strains was added to individual wells and cultivated at $37^{\circ} \mathrm{C}$ for $24-48 \mathrm{~h}$. Fluconazole concentrations, when reconstituted with the yeast suspensions, ranged from 0.12 to $64 \mu \mathrm{g} / \mathrm{ml}$. Chemical-free and yeast-free controls were included.

\section{Determination of minimum inhibitory and minimum fungicidal concentrations}

The minimum inhibitory concentration (MIC) of the evaluated compounds for each isolate was determined according to the CLSI M27-A3 methodology. The MIC was defined as the lowest concentration that produced a prominent decrease of fungal growth (inhibition $\geq 50 \%$ ) compared with growth control. The minimum concentration of drug that inhibited $90 \%$ and $50 \%$ of the isolates tested was defined as $\mathrm{MIC}_{90}$ and $\mathrm{MIC}_{50}$, respectively.

The MIC of fluconazole was read as the lowest concentration that produced a prominent decrease of growth $\left(\mathrm{MIC}_{2}\right.$ : inhibition $\left.\geq 50 \%\right)$ relative to the control of growth, with no antifungal agent. Classification of isolates in terms of their susceptibilities to these antifungal agents was based on the MIC breakpoints recommended in the M27-A3 protocol and the M27-S3 supplement of the CLSI [12]. The MIC for susceptibility was $\leq 8 \mu \mathrm{g} /$ 
$\mathrm{ml}$, the MIC for susceptible-dose dependent was 16 to $32 \mu \mathrm{g} / \mathrm{ml}$, and the MIC for resistance was $\geq 64 \mu \mathrm{g} / \mathrm{ml}$. For quality control, C. krusei ATCC 6258, and C. parapsilosis ATCC 22019, were used.

Minimum fungicidal concentration (MFC) determinations were performed according to the modifications suggested by Cantón et al. [13]. Fungicidal activity was defined as $a \geq 99.9 \%$ reduction in the number of colony-forming units from the starting inoculum count, whilst fungistatic activity was defined as $\leq 99.9 \%$ reduction. The minimum concentration of drug that is fungicidal to $90 \%$ of the isolates was defined as $\mathrm{MFC}_{90}$.

\section{Statistical analysis}

Unless otherwise specified, all tests were performed in triplicate in separate experiments. Descriptive statistics were used for MIC, MFC, the range and the geometric mean of these parameters. In vitro susceptibility testing data from $24 \mathrm{~h}$ and $48 \mathrm{~h}$ were compared for all natural compounds with the aim of detecting differences between both reading times. Comparisons between group values were performed by Student's t-test, being $\mathrm{p}$ $<0.05$ regarded as statistically significant.

\section{Results}

The MIC and MFC values of the 10 tested compounds against the 10 type strains studied at $24 \mathrm{~h}$ are summarized in Table 1. Table 2 shows MIC values of these compounds against oral isolates. The results confirmed the antifungal activity of almost all the tested compounds. The range of MICs at $24 \mathrm{~h}$ was $0.03-0.5 \%$ for carvacrol, $0.03-0.25 \%$ for the phenylpropanoid eugenol, $0.03-0.12 \%$ for geraniol, $0.03-0.25 \%$ for linalool, $0.06-0.5 \%$ for menthol, $0.06-4 \%$ for menthone, $0.06-0.5 \%$ for terpinen4-ol, $0.06-0.5 \%$ for $\alpha$-terpineol, and $0.25-32 \mu \mathrm{g} / \mathrm{ml}$ for fluconazole. Tween 80 at $0.05 \%(\mathrm{v} / \mathrm{v})$ did not show antifungal activity against the microorganisms studied (data not shown). Moreover, MIC readings at $48 \mathrm{~h}$ did not differ markedly from readings at $24 \mathrm{~h}$ ( $\mathrm{p}$ values were between 0.06 and 0.67 ). There were no differences over a dilution between the three measurements for each test, but the highest MIC was considered for calculations.

The most actives compounds against C. albicans were eugenol, geraniol, linalool and terpinen-4-ol with $\mathrm{MIC}_{50}$ $=0.12 \%$. The $\mathrm{MIC}_{50}$ of geraniol for C. tropicalis was $0.03 \%$ and for C. glabrata and C. guillermondii was $0.06 \%$. Eugenol and linalool were also very active against C. glabrata, C. guillermondii and C. tropicalis with $\mathrm{MIC}_{50}=0.06 \%$. Furthermore, terpinen-4-ol was also active against $C$. tropicalis with $\left(\mathrm{MIC}_{50}=0.06 \%\right)$, and $C$. glabrata and C. guillermondii $\left(\mathrm{MIC}_{50}=0.12 \%\right.$ for both species). In addition, carvacrol was also effective against C. glabrata and C. tropicalis $\left(\mathrm{MIC}_{50}=0.12 \%\right.$ for both species) and menthol against C. glabrata, C. guillermondii and C. tropicalis with $\mathrm{MIC}_{50}=0.12 \%$. The $\mathrm{MIC}_{50}$ of menthone for C. tropicalis was $0.06 \%$ and for C. glabrata was $0.12 \%$. These compounds were active against those species such as C. glabrata and C. krusei including isolates categorized as susceptible-dose dependent (2 or 5 out of 10 C. glabrata at 24 or 48 h, respectively) and resistant (1 C. krusei) to fluconazole.

Farnesol and tyrosol were the less potent evaluated compounds with range of MICs at $24 \mathrm{~h}$ of $0.12-8 \%$ and $0.5-8 \%$ respectively being farnesol more active against $C$. glabrata $\left(\mathrm{MIC}_{50}=0.25 \%\right)$ and $C$. dubliniensis $(\mathrm{MIC}=0.25 \%)$.

$\mathrm{MFC}_{90}$ values of the 10 tested compounds and fluconazole are also summarized in Table 2. Those compounds with more fungicidal activity against $C$. albicans were geraniol, carvacrol, eugenol, linalool and terpinen4-ol whose $\mathrm{MFC}_{90}$ ranged between 0.25 to $0.5 \%$.

\section{Discussion}

Essential oils are very complex natural mixtures extracted from several aromatic plants which can

Table 1 In vitro antifungal activity of the ten natural compounds against reference strains at 24 h (\%, v/v)

\begin{tabular}{|c|c|c|c|c|c|c|c|c|c|c|}
\hline & \multicolumn{10}{|c|}{ MIC/MFC } \\
\hline & Carvacrol & Eugenol & Farnesol & Geraniol & Linalool & Menthol & Menthone & Terpinen-4-ol & $\alpha$-terpineol & Tyrosol \\
\hline Candida albicans ATCC 90028 & $0.25 / 0.5$ & $0.12 / 0.12$ & $8 / 8$ & $0.12 / 0.12$ & $0.12 / 0.12$ & $0.25 / 0.5$ & $0.25 / 0.5$ & $0.12 / 0.12$ & $0.25 / 0.5$ & $4 / 8$ \\
\hline Candida albicans NCPF 3153 & $0.25 / 0.5$ & $0.06 / 0.12$ & $8 / 8$ & $0.06 / 0.12$ & $0.06 / 0.25$ & $0.25 / 0.5$ & $0.25 / 0.5$ & $0.06 / 0.12$ & $0.12 / 0.25$ & $8 / 8$ \\
\hline Candida albicans Ca2 & $0.25 / 0.5$ & $0.03 / 0.12$ & $1 / 8$ & $0.06 / 0.12$ & $0.06 / 0.12$ & $0.12 / 0.5$ & $0.12 / 0.5$ & $0.06 / 0.12$ & $0.12 / 0.25$ & $4 / 8$ \\
\hline Candida glabrata ATCC 90030 & $0.12 / 0.5$ & $0.03 / 0.12$ & $0.12 / 2$ & $0.12 / 0.12$ & $0.06 / 0.06$ & $0.12 / 0.5$ & $0.12 / 0.5$ & $0.12 / 0.12$ & $0.12 / 0.5$ & $8 / 8$ \\
\hline Candida glabrata NCPF 3203 & $0.25 / 0.25$ & $0.03 / 0.12$ & $0.12 / 0.5$ & $0.06 / 0.12$ & $0.12 / 0.25$ & $0.25 / 0.5$ & $0.25 / 0.5$ & $0.06 / 0.12$ & $0.12 / 0.5$ & $8 / 8$ \\
\hline Candida dubliniensis NCPF 3949 & $0.25 / 0.5$ & $0.06 / 0.12$ & $0.06 / 8$ & $0.06 / 0.12$ & $0.03 / 0.12$ & $0.12 / 0.25$ & $0.12 / 0.25$ & $0.12 / 0.5$ & $0.12 / 0.25$ & $4 / 8$ \\
\hline $\begin{array}{l}\text { Candida guilliermondii } \\
\text { NCPF3099 }\end{array}$ & $0.12 / 0.5$ & $0.03 / 0.06$ & $0.12 / 8$ & $0.06 / 0.06$ & $0.06 / 0.06$ & $0.12 / 0.25$ & $0.12 / 0.25$ & $0.06 / 0.12$ & $0.12 / 0.25$ & $4 / 8$ \\
\hline Candida krusei ATCC 6258 & $0.12 / 0.5$ & $0.12 / 0.5$ & $4 / 8$ & $0.12 / 0.5$ & $0.12 / 1$ & $0.5 / 2$ & $0.5 / 2$ & $0.06 / 0.25$ & $0.12 / 0.5$ & $2 / 4$ \\
\hline $\begin{array}{l}\text { Candida parapsilosis ATCC } \\
22019\end{array}$ & $0.06 / 0.5$ & $0.06 / 0.12$ & $8 / 8$ & $0.06 / 0.25$ & $0.06 / 0.5$ & $0.06 / 0.25$ & $0.06 / 0.25$ & $0.06 / 0.12$ & $0.06 / 0.5$ & $1 / 2$ \\
\hline Candida tropicalis NCPF3111 & $0.25 / 0.5$ & $0.12 / 0.12$ & $0.5 / 8$ & $0.06 / 0.12$ & $0.06 / 0.12$ & $0.12 / 0.5$ & $0.12 / 0.5$ & $0.12 / 0.12$ & $0.5 / 0.5$ & $8 / 8$ \\
\hline
\end{tabular}


Table 2 In vitro antifungal activity of the ten natural compounds against oral Candida isolates at $24 \mathbf{h}(\%, \mathbf{v} / \mathbf{v}) *$

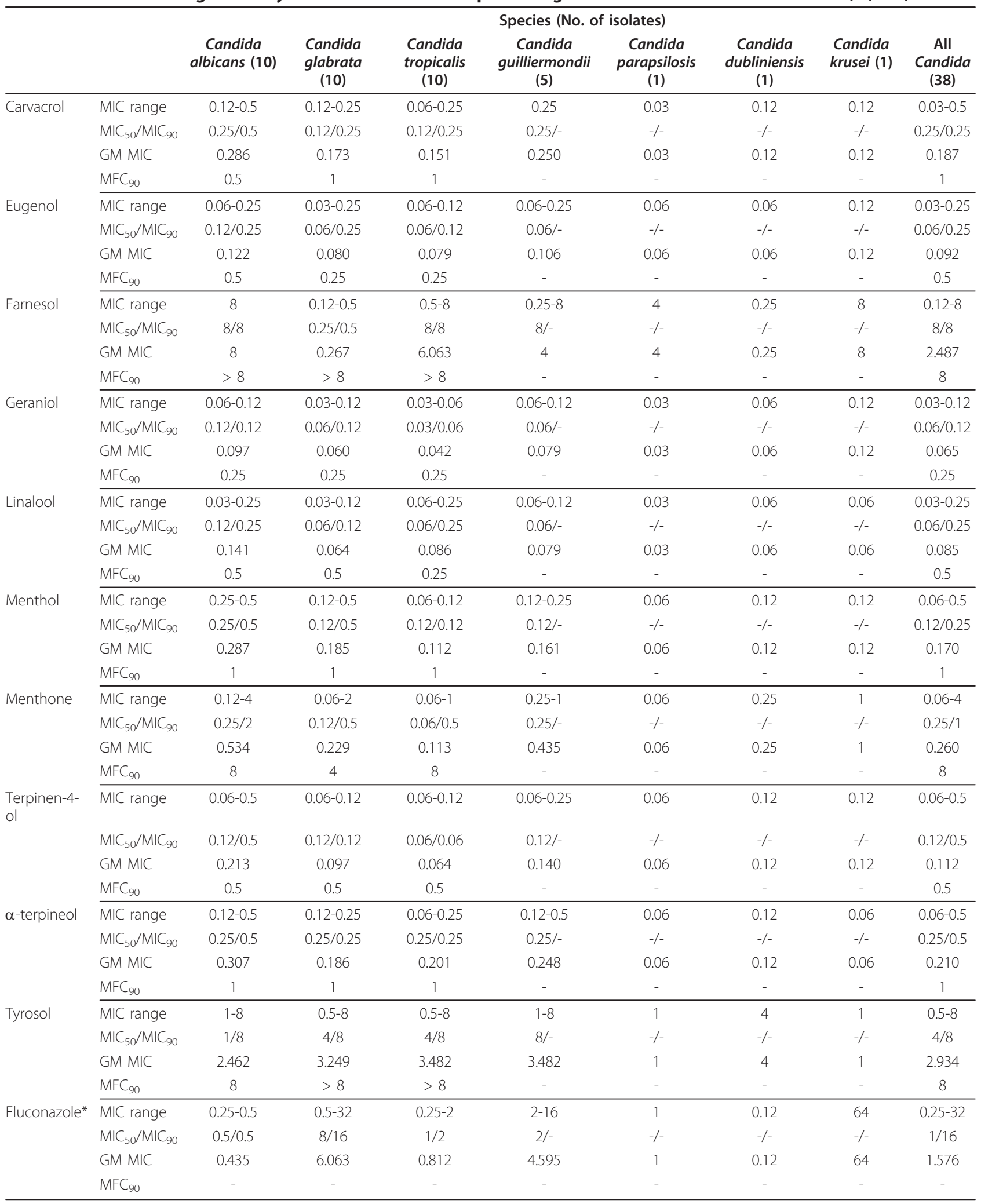

\footnotetext{
* Fluconazole concentrations are in $\mu \mathrm{g} / \mathrm{ml}$
} 
contain more than 20-60 components at quite different concentrations. The components are characterized by low molecular weight and include two groups of distinct biosynthetic origin. The main group is composed of terpenes and terpenoids. The second group includes aromatic and aliphatic products $[8,9]$. Terpenes are made from combinations of several 5-carbon-base units called isoprene. The monoterpenes are formed from the coupling of two isoprene units. They are the most representative molecules constituting $90 \%$ of the essential oils and allow a great variety of structures. Among the monoterpenes, geraniol and linalool are acyclic alcohols; menthol, terpinen-4-ol and $\mu$-terpineol monocyclic alcohols; menthones are monocyclic ketones; and carvacrol and thymol are phenols. The sesquiterpenes are formed from the assembly of three isoprene units, being the farnesol an alcohol [8]. The aromatic compounds such the eugenol, derived from phenylpropane, occur less frequently than the terpenes. Tyrosol is an antioxidant derivative of phenethyl alcohol present in a variety of natural sources such as the olive oil.

Many diverse activities of essential oils and their components have been shown such as antibacterial, antifungal, antioxidant, antitumor, analgesic and antioxidant activities [14]. Their antifungal properties are related to terpenes ability to pass through the fungal cell wall and locate between fatty acid chains of lipid bilayers, disrupting lipid packaging and altering the structure of the cell membrane [15,16]. Braga and Del Sasso [15] demonstrated by scanning electron microscopy that thymol affected the envelope of planktonic C. albicans. Changes in permeability and in membrane fluidity cause degradation of cell wall, a decrease in adherence to host's surfaces and variable effects such as disruption of cytoplasm membrane, leakage of cell contents, coagulation of cytoplasm and cell lyses $[8,9,14,17]$.

Compounds, such as carvacrol and geraniol, containing oxygen are frequently considered as those major responsible for this effect [8]. In some compounds, such as carvacrol and thymol, the mechanisms of action seem to be related to the inhibition of ergosterol biosynthesis [18].

When evaluating the antimicrobial properties of oils or their components, the method for determining MIC is important, in order to permit comparison of the data generated by different laboratories. For this reason, CLSI reference method for antifungal susceptibility testing was used in the current study [12], fluconazole was considered as comparator and the potential antifungal activity of almost all the tested components of the essential oils was observed. Eight of ten natural products: carvacrol, eugenol, geraniol, linalool, menthol, menthone, terpinen-4-ol, and $\alpha$-terpineol showed MICs ranged between $0.03 \%$ and $4 \%$. Conversely, farnesol and tyrosol showed lower antifungal activities with MICs ranging between $0.12 \%$ and $\geq 8 \%$. These latter compounds have been recognized as quorum-sensing molecules involved in the coordination of activities among groups of many single-celled organisms [19,20]. Farnesol and tyrosol are produced by $C$. albicans which block and accelerate, respectively, the morphological transition from yeasts to hyphae, and are important molecules in different steps of biofilm development and dispersion to other candidal foci during colonization and infection $[19,20]$. Their weak antifungal activities have been previously described by several authors [11,16]. However, He et al. [21] and Dalleau et al. [11] observed the high efficiency of some terpenes, such as carvacrol, eugenol geraniol and thymol, against C. albicans planktonic and sessile (biofilm) cells.

The phenylpropanoid eugenol and the oxygenated monoterpenes geraniol, linalool and terpinen-4-ol were the compounds with the strongest antifungal activity against $C$. albicans. Other authors have observed the potent antifungal or even fungicidal effects of $\alpha$-terpineol and terpinen-4-ol [17,22,23].

Carvacrol is an important component of several essential oils from plants, such as Origanum spp., Satureja hortensis, Thymus spp. and Thymbra capitata. In agreement with our observations, other authors have found that this compound exhibited a potent anti-Candida activity $[11,24]$ even against fluconazole-resistant Candida isolates. In the current study, carvacrol and the rest of tested compounds, with the exception of farnesol, menthone and tyrosol, showed important in vitro activities at very low concentrations against the fluconazoleresistant C. krusei and the susceptible-dose dependent C. glabrata isolates. Moreover, carvacrol was able to inhibit in vitro germ tube formation and filamentation of C. albicans [8]. Tampieri et al. [16] considered that carvacrol and geraniol were the most active against $C$. albicans as a concentration of 100 ppm of these compounds inhibited the growth of the unique strain tested.

Some studies have shown that primary monoterpenic alcohols (geraniol and citronellol) have more potent antifungal activity than tertiary alcohols (linalool). Linalool activity is controversial as some authors consider this compound inactive while others found a moderate antifungal activity $[8,16]$. However, in the current study, we have observed a strong fungicidal activity in both geraniol as linalool $\left(\mathrm{MFC}_{90} 0.25 \%\right.$ for geraniol and $0.5 \%$ for linalool).

Mondello et al. (2006) reported that terpinen-4-ol (main component of Melaleuca alternifolia -tea tree-oil) was fungistatic $\left(\mathrm{MIC}_{90}\right.$ of $\left.0.06 \%\right)$ and fungicidal $\left(\mathrm{MFC}_{90}\right.$ of $0.125 \%$ ) against fluconazole-susceptible and resistant C. albicans isolates. These authors suggested that this compound could be the mediator of the in vivo activity 
of tea tree oil in a rat model of vulvovaginal candidiasis. Similar antifungal activities of terpinen-4-ol has been observed in the present study, and MIC ranged from 0.06-0.12\% for C. parapsilosis and C. tropicalis, to 0.06$0.5 \%$ for C. albicans. Finally, the phenylpropanoid eugenol, main component of cinnamon and clove oils, as in our study, is considered to have a potent anti-Candida activity $[8,25]$. We should stress that there are important difficulties to compare the current results with those reported by other authors due to the different in vitro susceptibility tests used and the potential variation of susceptibilities among the isolates and strains studied. Moreover, many studies have tested C. albicans or a very limited number of fungal species or even a unique isolate of each species $[16,26,27]$.

On the other hand, the topical use of these naturally occurring plant active principles is relatively safe, and their side effects reported in the literature are minor, self-limiting and occasional $[14,24,28,29]$. Terpenes have the potential to be toxic if ingested at higher doses and can also cause skin irritation at higher concentrations. However, low doses of carvacrol or terpinen-4-ol did not induced toxicity for the mucosa in rat models of Candida vaginitis [24,28]. Moreover, a big advantage of essential oils and their components is the fact that they are usually devoid of long-term genotoxic risks [14,29].

\section{Conclusions}

Some natural compounds from essential oils, such as carvacrol, eugenol, geraniol, linalool and terpinen-4-ol were active in vitro against a collection of oral Candida isolates. Their fungistatic and fungicidal activities might convert them into promising alternatives for the topic treatment of oral candidiasis and Candida-associated denture stomatitis. However, potential intolerance and/ or toxic effects of some of these compounds should be taken into consideration.

\section{Acknowledgements}

Written consent for publication was obtained from the patients. Funding: this work has been funded in part by projects GIC07 123-IT-222-07 (Departamento de Educación, Universidades e Investigación, Gobierno Vasco), S-PRO9UNO1 and S-PR10UN03 (Saiotek 2009 and 2010, Departamento de Industria, Comercio y Turismo, Gobierno Vasco).

\section{Authors' contributions}

CMA, EE and GQ designed the study. CMA, LM and EE collected the data. CMA and EE performed the techniques employed in the study. CMA, EE and LM made the interpretation of statistical analyses. EE and GQ wrote the paper with input from all the authors who each approved the final version.

\section{Competing interests}

The authors declare that they have no competing interests.

Received: 30 June 2011 Accepted: 26 November 2011 Published: 26 November 2011

\section{References}

1. Gendreau L, Loewy ZG: Epidemiology and etiology of denture stomatitis. J Prosthodont 2011, 20:251-260.

2. Marcos-Arias C, Vicente JL, Sahand IH, Eguía A, De-Juan A, Madariaga L, Aguirre JM, Eraso E, Quindós G: Isolation of Candida dubliniensis in denture stomatitis. Arch Oral Biol 2009, 54:127-131.

3. Samaranayake LP, Leung WK, Jin L: Oral mucosal fungal infections. Periodontology 2000 2009, 49:39-59.

4. Webb BC, Thomas CJ, Willcox MD, Harty DW, Knox KW: Candida-associated denture stomatitis. Aetiology and management: a review. Part 1. Factors influencing distribution of Candida species in the oral cavity. Aust Dent J 1998, 43:45-50

5. Webb BC, Thomas CJ, Willcox MD, Harty DW, Knox KW: Candida-associated denture stomatitis. Aetiology and management: a review. Part 3. Treatment of oral candidosis. Aust Dent J 1998, 43:244-249.

6. Barchiesi F, Silvestri C, Arzeni D, Ganzetti G, Castelletti S, Simonetti O, Cirioni O, Kamysz W, Kamysz E, Spreghini E, Abruzzetti A, Riva A, Offidani AM, Giacometti A, Scalise G: In vitro susceptibility of dermatophytes to conventional and alternative antifungal agents. Med Mycol 2009, 47:321-326.

7. Hammer KA, Carson CF, Riley TV: Melaleuca alternifolia (tea tree) oil inhibits germ tube formation by Candida albicans. Med Mycol 2000 38:355-362.

8. Palmeira de Oliveira A, Salgueiro L, Palmeira de Oliveira R, Martinez de Oliveira J, Pina Vaz C, Queiroz JA, Rodrigues AG: Anti-Candida activity of essential oils. Mini-Reviews in Medicinal Chemistry 2009, 9:1292-1305.

9. Pauli A: Anticandidal low molecular compounds from higher plants with special reference to compounds from essential oils. Med Res Rev 2006 26:223-268.

10. Eraso E, Moragues MD, Villar-Vidal M, Sahand IH, González-Gómez N, Pontón J, Quindós G: Evaluation of the new chromogenic medium Candida ID 2 for isolation and identification of Candida albicans and other medically important Candida species. J Clin Microbiol 2006, 44:3340-3345.

11. Dalleau $S$, Cateau $E$, Bergès $T$, Berjeaud JM, Imbert $C$ : In vitro activity of terpenes against Candida biofilms. Int J Antimicrob Agents 2008, 31:572-576.

12. Clinical and Laboratory Standards Institute: Reference method for broth dilution antifungal susceptibility testing of yeasts; approved standard. 3 edition. CLSI, Wayne, PA, USA; 2010, CLSI document M27-A3 and Supplement S3.

13. Cantón E, Pemán J, Viudes A, Quindós G, Gobernado M, Espinel-Ingroff A: Minimum fungicidal concentrations of amphotericin $B$ for bloodstream Candida species. Diagn Microbiol Infect Dis 2003, 45:203-206.

14. Bakkali F, Averbeck S, Averbeck D, Idaomar M: Biological effects of essential oils-a review. Food Chem Toxicol 2008, 46:446-475.

15. Braga PC, Culici M, Alfieri M, Dal Sasso M: Thymol inhibits Candida albicans biofilm formation and mature biofilm. Int J Antimicrob Agents 2008, 31:472-477.

16. Tampieri MP, Galuppi R, Macchioni F, Carelle MS, Falcioni L, Cioni PL, Morelli I: The inhibition of Candida albicans by selected essential oils and their major components. Mycopathologia 2005, 159:339-345.

17. Cox SD, Mann CM, Markham JL, Bell HC, Gustafson JE, Warmington JR, Wyllie SG: The mode of antimicrobial action of the essential oil of Melaleuca alternifolia (tea tree oil). J Appl Microbiol 2000, 88:170-175.

18. Ahmad A, Khan A, Akhtar F, Yousuf S, Xess I, Khan LA, Manzoor N: Fungicidal activity of thymol and carvacrol by disrupting ergosterol biosynthesis and membrane integrity against Candida. Eur J Clin Microbiol Infect Dis 2011, 30:41-50.

19. Alem MA, Oteef MD, Flowers TH, Douglas LJ: Production of tyrosol by Candida albicans biofilms and its role in quorum sensing and biofilm development. Eukaryot Cell 2006, 5:1770-1779.

20. Ramage G, Saville SP, Wickes BL, López-Ribot JL: Inhibition of Candida albicans biofilm formation by farnesol, a quorum-sensing molecule. Appl Environ Microbiol 2002, 68:5459-5463.

21. He M, Du M, Fan M, Bian Z: In vitro activity of eugenol against Candida albicans biofilms. Mycopathologia 2007, 163:137-143.

22. Oliva B, Piccirilli E, Ceddia T, Pontieri E, Aureli P, Ferrini AM: Antimycotic activity of Melaleuca alternifolia essential oil and its major components. Lett Appl Microbiol 2003, 37:185-187. 
23. Traboulsi RS, Mukherjee PK, Ghannouma MA: In vitro activity of inexpensive topical alternatives against Candida spp. isolated from the oral cavity of HIV-infected patients. Int J Antimicrob Agents 2008, 31:272-276.

24. Chami F, Chami N, Bennis S, Trouillas J, Remmal A: Evaluation of carvacrol and eugenol as prophylaxis and treatment of vaginal candidiasis in an immunosuppressed rat model. J Antimicrob Chemother 2004, 54:909-914.

25. Ahmad A, Khan A, Khan LA, Manzoor N: In vitro synergy of eugenol and methyleugenol with fluconazole against clinical Candida isolates. $J$ Med Microbiol 2010, 59:1178-1184

26. Chao SC, Young DG, Oberg CJ: Screening for inhibitory activity of essential oils on selected bacteria, fungi and viruses. J Essential Oil Res 2000, 12:639-649.

27. Concha JM, Moore LS, Holloway WJ: Antifungal activity of Melaleuca alternifolia (tea-tree) oil against various pathogenic organisms. J Am Podiatr Med Assoc 1988, 88:489-492.

28. Mondello F, De Bernardis F, Girolamo A, Cassone A, Salvatore G: In vivo activity of terpinen-4-ol. The main bioactive component of Malaleuca alternifolia Cheel (tea tree) oil against azole-susceptible and -resistant human pathogenic Candida species. BMC Infect Dis 2006, 6:158.

29. Hammer KA, Carson CF, Riley TV, Nielsen J: A review of the toxicity of Melaleuca alternifolia (tea-tree oil). Food Chem Toxicol 2006, 44:616-625.

\section{Pre-publication history}

The pre-publication history for this paper can be accessed here: http://www.biomedcentral.com/1472-6882/11/119/prepub

doi:10.1186/1472-6882-11-119

Cite this article as: Marcos-Arias et al: In vitro activities of natural products against oral Candida isolates from denture wearers. BMC Complementary and Alternative Medicine 2011 11:119.

\section{Submit your next manuscript to BioMed Central and take full advantage of:}

- Convenient online submission

- Thorough peer review

- No space constraints or color figure charges

- Immediate publication on acceptance

- Inclusion in PubMed, CAS, Scopus and Google Scholar

- Research which is freely available for redistribution

Submit your manuscript at www.biomedcentral.com/submit
C Biomed Central 